\section{SAÚDE E AMBIENTE}

V.8 • N.3 • 2021 - Fluxo Contínuo

ISSN Digital: 2316-3798

ISSN Impresso: 2316-3313

DOI: 10.17564/2316-3798.2021v8n3p151-162
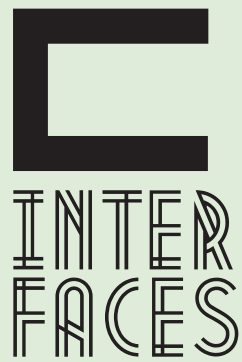

CIENTÍFICAS

\title{
PARASITOSES EM CÃES DOMICLLIADOS EM UM MUNICÍPIO DO SUL DO BRASIL
}

PARASITIC DISEASES IN HOUSEHOLD DOGS IN A MUNICIPALITY IN SOUTHERN BRAZIL

PARASITOSES EN PERROS DOMÉSTICOS EN UN MUNICIPIO DEL SUR DE BRASIL

Jéssica Moreira de Paula ${ }^{1}$ Veridiana Simon ${ }^{2}$

Dárquila Regina Ladik Piva ${ }^{3}$

Thainá Terras Kemerich ${ }^{4}$ Flávia Damolin ${ }^{5}$ Douglas Michel Muller Fritzen ${ }^{6}$ Junir Antônio Lutinski

\section{RESUMO}

Parasitos podem causar danos à saúde de animais de companhia e podem afetar a saúde humana. Este estudo teve como objetivo investigar a prevalência e a diversidade de parasitoses em cães domiciliados em um município do sul do Brasil. As coletas ocorreram nos meses de outubro a dezembro de 2018, em três bairros da cidade. Um animal por residência foi incluído na amostra, totalizando 150. Para a avaliação de ectoparasitas foram utilizados os meios semiológicos de investigação. Foram coletadas fezes frescas dos animais para a busca por endoparasitas. As amostras foram conduzidas e avaliadas no Laboratório de Zoologia e Parasitologia da Unochapecó. Para o processamento das amostras foram utilizadas duas técnicas, sendo a de flutuação (Millis-Molay) e sedimentação (Dennis Stones). 0 estudo contou ainda com um inquérito junto aos tutores dos animais sobre as condições de bem-estar e cuidados com a saúde deles. Ao todo, em 23 cães (15,3\%) foram encontrados ectoparasitas da espécie Ctenocephalides canis. Foram observados os endoparasitas Trichuris vulpis (6,0\% pela técnica de flutuação e 3,3\% por sedimentação) e Ancylostoma spp. (4,7\%) pela técnica de flutuação. A frequência de animais acometidos por parasitos destaca a importância de cuidados mais amplo e sistemático com a saúde dos animais.

\section{PALAVRAS-CHAVE}

Saúde Animal. Saúde Pública. Vigilância em Saúde. 


\section{ABSTRACT}

Parasites can cause damage to the health of pets and can affect human health. This study aimed to investigate the prevalence and diversity of parasites in dogs domiciled in a municipality in southern Brazil. The collections took place from October to December 2018, in three neighborhoods of the city. One animal per household was included in the sample, totaling 150 . For the evaluation of ectoparasites, the semiological means of investigation were used. Fresh animal feces were collected to search for endoparasites. The samples were conducted and evaluated at the Unochapecó Zoology and Parasitology Laboratory. To process the samples, two techniques were used: floating (Millis-Molay) and sedimentation (Dennis Stones). The study also included a survey with animal tutors about their welfare and health care conditions. In all, 23 dogs (15.3\%) presented ectoparasites specie of Ctenocephalides canis (Curtis, 1826). The endoparasites found were Trichuris vulpis $(6.0 \%$ by flotation technique and $3.3 \%$ by sedimentation) and Ancylostoma spp. ( $4.7 \%)$ by the flotation technique. The frequency of animals affected by parasites highlights the importance of broader and systematic animal health care.

\section{KEYWORDS}

Animal health. Public health. Health surveillance.

\section{RESUMEN}

Los parásitos pueden dañar la salud de las mascotas y afectar la salud humana. Este estudio tuvo como objetivo investigar la prevalencia y diversidad de parásitos en perros domiciliados en un municipio del sur de Brasil. Las investigaciones se llevaron a cabo de octubre a diciembre de 2018, en tres barrios de la ciudad. En la muestra se incluyó un animal por residencia, totalizando 150. Para la evaluación de ectoparásitos se utilizaron medios de investigación semiológicos. Se recogieron heces frescas de los animales para la búsqueda de endoparásitos. Las muestras fueron evaluadas en el Laboratorio de Zoología y Parasitología de Unochapecó. Para el procesamiento de las muestras se utilizaron dos técnicas: flotación (Millis-Molay) y sedimentación (Dennis Stones). El estudio también incluyó una encuesta con tutores de animales sobre su bienestar y condiciones de atención médica. En total, en 23 perros (15,3\%) se encontraron ectoparásitos de la especie Ctenocephalides canis. Los endoparásitos Trichuris vulpis (6,0\% por la técnica de flotación y 3,3\% por sedimentación) y Ancylostoma spp. (4,7\%) utilizando la técnica de flotación. La frecuencia de animales afectados por parásitos destaca la importancia de una atención más amplia y sistemática para la salud de los animales. 


\section{PALABRAS CLAVE}

Bienestar animal, Sanidad animal, Salud pública, Vigilancia de la salud.

\section{INTRODUCÇÃO}

O crescimento populacional de animais domésticos é observado no mundo todo, sendo cães a espécie de convívio mais próxima com o ser humano (RIBEIRO et al., 2015). Esta relação pode oferecer benefícios psicológicos, fisiológicos e sociais aos seres humanos (BARNABE et al., 2015; BARROS et al., 2018). A sensibilidade desses animais para compreender o mundo à sua volta, inclusive os sentimentos dos seres ao seu redor, permite que sejam candidatos potenciais para trabalhos de reabilitação de pacientes humanos. Contudo, o contato direto com cães impõe aos tutores a necessidade de um cuidado rotineiro com a saúde do animal, uma vez que quando os cuidados são inadequados, podem representar uma fonte de infecção e de transmissão de agentes patogênicos, por exemplo, espécies de ectoparasitas e de endoparasitas (BARROS et al., 2018).

Os ectoparasitas de maior prevalência em cães são as pulgas do gênero Ctenocephalides Stiles e Collins, 1930 (Insecta, Siphonaptera) e carrapatos do gênero Rhipicephalus Koch, 1844 (Acari, Ixodida). Os endoparasitas com maiores prevalências em cães pertencem aos gêneros Ancylostoma Dubini, 1843 (Nematoda, Strongylida) e Toxocara Stiles, 1905 (Nematoda, Ascaridida) (ARAÚJO, 2015; BARNABE et al., 2015; RIBEIRO et al., 2015). As pulgas (Ctenocephalides) na fase adulta, são hematófagos e têm preferência por determinado hospedeiro. Esses insetos podem hospedar agentes como Dipylidium caninum (Linnaues, 1758), Hymenolepis nana [syn. Rodentolepis nana (von Siebold, 1852)] e Hymenolepis diminuta (Rudolphi, 1819) (Cestoda, Cyclophyllidea).

As pulgas vivem parte do tempo sobre o corpo dos hospedeiros e parte no ambiente, onde se desenvolvem os ovos, as larvas e as pupas. Podem se alimentar de 2 a 3 vezes ao dia. A picada pode produzir dermatite, desenvolvendo-se na pele lesão eritematosa e edemaciada, às vezes com formação de nódulo endurecido ou pústula (OLIVEIRA et al., 2008). Os carrapatos podem ser encontrados na natureza e em seus hospedeiros as fases de larvas, ninfas ou adultos. São hematófagos e podem causar agravos à saúde animal ou humana como a escabiose, Doença de Lyme, Febre Maculosa, Babesiose e Anaplasmose (YOSHINARI et al., 2010).

Ectoparasitas como insetos e ácaros podem ser transmitidos de forma direta pelo contato dos cães com humanos. Os ovos dos parasitos intestinais são eliminados pelas fezes dos animais parasitados, contaminando o ambiente e proporcionando a transmissão para outros hospedeiros (RIBEIRO et al., 2015; BARROS et al., 2018). Parasitos com potencial zoonótico impactam a saúde e o bem-estar dos animais de companhia e a saúde humana além da saúde dos animais. Muitos estão associados às condições de saneamento básico inadequado (LALLO et al., 2016).

As parasitoses podem desencadear reações inflamatórias, alérgicas e, em casos mais graves, quadros anêmicos especialmente em crianças. Em humanos desencadeiam complicações já conhecidas 
como o baixo rendimento escolar de crianças e adolescentes, baixa produtividade no trabalho em adultos e aumento de gastos com a saúde (CHIEFFI et al., 2002). A ocorrência de parasitoses intestinais em humanos é encontrada em determinadas localidades que dependem das interações entre hospedeiros, parasitos e o ambiente compartilhado.

Alguns tipos de parasitoses como ancilostomíase, escabiose, toxoplasmose, leishmaniose e leptospirose podem tornar-se potencialmente graves se não controladas (CHIEFFI; NETO, 2003). Contudo, a prevalência de parasitos (ectoparasitas e endoparasitas) em animais domésticos (GONZÁLEZ; GIRALDO, 2015) como cães é ignorada em muitos casos, assim como o impacto desses agentes sobre a saúde e bem-estar animal.

Nesse contexto, o presente trabalho teve como objetivo investigar como a prevalência e a diversidade de parasitoses em cães domiciliados em um município do sul do Brasil.

\section{METODOLOGIA}

\subsection{CARACTERIZAÇÃO E LOCAL DO ESTUDO}

Trata-se de uma pesquisa de caráter quantitativo transversal. 0 estudo foi realizado no município de Chapecó/SC ( $27^{\circ} 5^{\prime} 48^{\prime \prime}$; $\left.52^{\circ} 37^{\prime} 7^{\prime \prime} \mathrm{W}\right)$. Com o objetivo de contemplar uma área geográfica maior da cidade, três bairros foram incluídos na pesquisa, sendo: Bom Pastor, Efapi e Santa Maria. Essa seleção deu-se com base no plano de desenvolvimento e zoneamento do Município de Chapecó (CHAPECÓ, 2019).

\subsection{AMOSTRA}

Adotou-se a amostragem sistemática preconizada por Vieira e colaboradores (2019). Foram sorteados 50 quarteirões em cada bairro, sendo que em cada quarteirão foi contemplado um domicílio residencial. 0 sorteio dos quarteirões foi efetuado utilizando-se o software Excel for Windows. Como critérios de inclusão foram adotados: a) haver pelo menos um cão domiciliado no imóvel; b) estar presente, no momento da visita, o tutor do(s) animal(is) (pessoa com mais de 18 anos de idade que se autodeclarou responsável pelos animais). Apenas um animal foi avaliado por domicílio, totalizando 50 por bairro, 150 no total. Incluiu-se no estudo um cão por domicílio. A escolha do animal domiciliado deu-se mediante sorteio, caso fosse encontrado mais de um. Como critério de exclusão, caso o imóvel era desconsiderado da amostra e o seguinte era incluído.

\subsection{COLETA DOS DADOS}

As amostras foram obtidas no período entre os meses de outubro e dezembro de 2018. Para a avaliação de ectoparasitas foram utilizados meios semiológicos descritos por Feitosa (2016), que incluem a palpação, olfação, inspeção direta e indireta. Para a realização do exame físico foi necessária à contenção física do animal, mediante a utilização de focinheira e cambão ou o auxílio do tutor caso o animal fosse de pequeno porte e dócil. 
De acordo com Monteiro (2017), na pesquisa de ectoparasitas, no caso da escabiose, faz-se um raspado cutâneo com uma lâmina até ocorrer leve sangramento. Posteriormente, acondiciona-se a amostra em uma lâmina para leitura em microscópio estereoscópio. Piolhos, pulgas e ácaros faz-se coleta manual com algodão embebido em álcool. Para retirar carrapatos deve segurar no ponto de fixação na pele e girar levemente antes de tracionar. As amostras obtidas foram identificadas e transportadas ao Laboratório de Zoologia e Parasitologia da Unochapecó, para identificação.

Foram coletadas amostras de fezes frescas dos animais (que tinham sido evacuadas pelo animal durante o período em que os pesquisadores estiveram no domicílio ou nas quatro horas que antecederam a chegada dos pesquisadores, segundo relatos dos proprietários). As amostras foram acondicionadas em potes específicos, identificadas com o número da coleta, nome do animal e tutor, data e endereço. Posteriormente, encaminhadas ao Laboratório de Zoologia e Parasitologia da Unochapecó. As amostras foram processadas, utilizando dois métodos coproparasitológicos: Método de Flutuação (Willis-Molay) e Método de Sedimentação (Denis Swanson Stone). A identificação das espécies de ectoparasitas e endoparasitas seguiu os guias propostos por Urquhart e colaboradores (1988) e Monteiro (2017).

Adicionalmente, aos tutores, foi aplicado um questionário abordando conhecimentos sobre zoonoses, formas de medicina veterinária preventiva e saúde pública. Foram abordadas questões como a frequência de vacinação e vermifugação dos animais, assim como sobre a busca por informações para a manutenção dos mesmos. As questões foram previamente estruturadas, sendo todas objetivas. As respostas foram registradas pelos pesquisadores em formulários impressos e transcritos para um banco de dados.

\subsection{ANÁLISE DOS DADOS}

Os dados foram registrados em um banco de dados e a avaliação foi feita a partir de análises de frequência das respostas. Foram utilizadas estatísticas descritivas (média e desvio padrão) para caracterizar e comparar as respostas obtidas. Para tal, foi utilizado o software Excel for Windows. Foi utilizado o pré-teste Shapiro-Wilk para testar a normalidade das variáveis quantitativas. Para comparar os grupos foram utilizados os testes de Mann-Whitney (U) e de Kruskal-Wallis (Hc). Foi utilizada significância estatística "p" < 0,05. Os testes foram realizados com o auxílio do software Past.

\subsection{ASPECTOS ÉTICOS}

Todos os princípios éticos foram respeitados, preservando a identidade dos participantes e não oferecendo nenhum dano à integridade física ou psicológica deles. A pesquisa foi aprovada pelo CEUA Unochapecó sob número 005-18 e pelo CEP/CONEP sob número 2.880.004. 


\section{RESULTADOS}

Em um total de 23 cães (15,3\%) observou-se a presença do ectoparasito Ctenocephalides canis (Curtis, 1826). Ao todo, foram encontrados sete cães com lesões compatíveis para a realização de raspados cutâneos, contudo, todos apresentaram resultado negativo para escabiose.

A avaliação coproparasitológica por flutuação resultou em 12,7\% de amostras positivas. Os endoparasitas encontrados foram os nematódeos Ancylostoma caninum (Ercolani, 1859) (4,7\%) e Trichuris vulpis (Frölich, 1789) (6,0\%). No método de sedimentação, 3,3\% das amostras estavam positivas para a presença de Trichuris vulpis

Ao todo, $70,67 \%$ dos tutores declarou não fazer uso de vermífugos. Um total de $10,67 \%$ faz uso duas vezes ao ano e $18,67 \%$ apenas uma vez. Quanto ao conhecimento sobre zoonoses, $80,67 \%$ declararam conhecer o significado da palavra. Quando questionados sobre a fonte das informações sobre o tema zoonoses, 37,33\% responderam ter assistido na televisão e 32,67\% a partir de conversas informais com amigos ou profissionais de saúde. Sites da internet foram referidos como fonte para $10 \%$ dos tutores.

A média verificada do número de cães domiciliados que recebem vermífugos foi significativamente maior do que a média dos que não receberam. 0 número médio de animais que recebem vermífugos a cada quatro meses ou a cada seis meses também é maior do que aqueles que recebem apenas uma vez por ano. 0 número médio de cães que recebem ectoparasitários não difere da média daqueles não o recebem. A frequência de banhos uma vez por semana foi a mais encontrada (TABELA 1).

Tabela 1 - Medidas preventivas adotadas por tutores de cães domiciliados na cidade de Chapecó, SC, 2018

\begin{tabular}{|c|c|c|c|}
\hline & Média & Desvio padrão & Significância \\
\hline \multicolumn{4}{|c|}{ Cães que recebem vermífugos* } \\
\hline Sim & 1,25 & 1,10 & \multirow{2}{*}{$\begin{array}{l}U=6681 \quad p< \\
0,0001\end{array}$} \\
\hline Não & 0,51 & 0,90 & \\
\hline \multicolumn{4}{|c|}{ Frequência com que os cães recebem vermífugos** } \\
\hline A cada quatro meses & 0,55 & 0,96 & \multirow{3}{*}{$\begin{array}{l}H c=12,8 \\
p=0,001\end{array}$} \\
\hline A cada seis meses & 0,46 & 0,94 & \\
\hline Uma vez ao ano & 0,21 & 0,60 & \\
\hline \multicolumn{4}{|c|}{ Cães que recebem antipulga* } \\
\hline Sim & 0,97 & 1,13 & \multirow{2}{*}{$U=10444 ; p=0,24$} \\
\hline Não & 0,79 & 0,99 & \\
\hline \multicolumn{4}{|c|}{ Frequência com que os cães recebem banho** } \\
\hline
\end{tabular}




\begin{tabular}{llll}
\hline & Média & Desvio padrão & \multicolumn{1}{c}{ Significância } \\
\hline Uma vez por semana & 0,84 & 1,05 & \\
Duas vezes por semana & 0,44 & 0,76 & $\mathrm{Hc}=65,07 ; \mathrm{p}<$ \\
Uma vez por mês & 0,27 & 0,74 & 0,0001 \\
Nunca & 0,21 & 0,62 & \\
\hline
\end{tabular}

* teste de Mann-Whitney (U); **Teste Kruskal-Wallis (Hc).

Fonte: Dados da pesquisa

\section{DISCUSSÃO}

A prevalência de ectoparasitas e de endoparasitas nos cães amostrados foi relativamente baixa $(15,3 \%)$ quando comparada aos resultados encontrados por Stalliviere e colaboradores (2009) em outro município catarinense (22,9\%). 0 mesmo foi observado quanto à diversidade dos parasitos. Os resultados quanto à prevalência das parasitoses encontram suporte nos relatos dos tutores sobre os cuidados adotados com a saúde e com o bem-estar nos animais. O cuidado por parte dos tutores para com os cães domiciliados contribui para a incidência de parasitos (FERRAZ et al., 2019).

A presença de ectoparasitos ficou restrita a uma espécie, Ctenocephalides canis. Segundo Oliveira e colaboradores (2008), Ctenocephalides canis e Ctenocephalides felis (Bouche, 1835) são encontradas com frequência em cães e gatos, respectivamente. Os sinais clínicos nos animais se caracterizam por alergias à picada que pode desencadear irritação, causando desconforto. Dentre os animais identificados com lesões cutâneas, somente em dois foram encontradas pulgas.

A infecção por helmintos e enteroprotozoários pode ocorrer pela ingestão de água e alimentos contaminados com cistos ou ovos ou ainda pela penetração de larvas pela pele ou mucosas (MOTTA; SILVA, 2002). O hospedeiro pode também disseminar os parasitos para o ambiente, para outros animais ou para o ser humano (CHIEFFI et al., 2002). Como medidas de prevenção, faz-se necessário o uso de medidas de higiene, visando minimizar a transmissão das parasitoses. Segundo Chieffi e colaboradores (2002), o diagnóstico pode ser realizado por meio de exames parasitológicos de fezes (EPF) e pelos cuidados com os animais de convívio. 0 acompanhamento periódico do animal por um profissional Médico Veterinário se faz necessário para prevenir estas e outras doenças que acometem cães e o ser humano (MONTEIRO, 2017).

Os helmintos estão entre os agentes etiológicos frequentemente transmitidos entre animais de convívio e o ser humano (MONTEIRO, 2017). Nos animais, a infecção pode ocorrer pela ingestão da larva infectante, bem como pela penetração cutânea. Nas duas vias, as larvas chegam ao intestino e se aderem à mucosa intestinal. Os sintomas mais frequentes são anemia, fezes sanguinolentas ou escuras, perda de peso e abdômen abaulado (MARMOL, 2018). 0 controle e a profilaxia dessa parasitose devem considerar as condições de sanidade dos animais, everminação periódica, a procedência dos animais e acesso à rua e locais públicos, a fim de evitar a contaminação do ambiente e do ser humano (LIMA, 2017). 
Trichuris vulpis é um helminto de cães que facilmente pode ser identificado em outros mamíferos silvestres. A infecção ocorre pela ingestão dos ovos infectantes (TROCCAP, 2017) e infecções intensas podem causar diarreia (MOTTA; SILVA, 2002). Frequentemente esse parasito acomete os cães após o desmame (GENNARI, 2015). Para cães que vivem em um ambiente contaminado, os ovos são uma fonte de infecção. Cães adultos apresentam uma carga parasitária maior quando comparado aos animais jovens (TRAVERSA, 2011).

Observou-se no estudo que $18,67 \%$ dos tutores fazem uso anualmente de vermífugos. A frequência do uso de vermífugos é determinada pela exposição do cão a ambientes contaminados (RIBEIRO, 2004) portanto, cães com acesso à rua e que tenham contato com outros cães necessitam de uma maior atenção. Os resultados apontam que a maioria não faz uso de vermífugos, deixando os animais mais suscetíveis à infecção por parasitoses.

Dentre os tutores, aqueles que trabalham nas agroindústrias da cidade relataram que anualmente são obrigados a fazer exame coproparasitológico e, no caso de confirmação de parasitismo fazem uso de vermífugos. Segundo Chieffi e Neto (2003), as doenças parasitárias intestinais podem ser tratadas por meio de doses únicas ou de medicamentos com amplo espectro de atividade. Isso reduz a contaminação em humanos e no ambiente, resultando na proteção da saúde dos animais.

Ao todo, mais de $80 \%$ dos tutores já ouviu falar sobre o tema zoonoses, um percentual maior do que o encontrado (73\%) por Olivari e colaboradores (2015) em um município do estado de São Paulo. Os meios pelos quais as informações chegam à população são variados, contudo, canais de comunicação considerados mais abrangentes como a internet ainda representam baixo poder alcance, comparado aos meios tradicionais como a televisão. Tratando-se de um tema relacionado à saúde pública e ao bem-estar animal, o conhecimento pela maioria dos tutores é um achado importante tanto para a saúde animal quanto para fundamentar estratégias de controle de zoonoses (KATAGIRI; OLIVEIRA-SEQUEIRA, 2007). Contudo, os canais de comunicação usados para a divulgação de informações podem ser reavaliados.

Quanto às medidas preventivas, o uso e a frequência de vermífugos e antipulgas, a maioria dos cães recebe tais cuidados, segundo os tutores. Por outro lado, $100 \%$ dos cães incluídos na pesquisa nunca tiveram um exame coproparasitológico antes deste estudo. 0 uso desnecessário de antiparasitários pode apresentar consequências indesejáveis para a saúde dos animais como perturbações do trato gastrointestinal, além de desenvolver resistência parasitária (KATAGIRI; OLIVEIRA-SEQUEIRA, 2007).

\section{CONCLUSÃO}

A prevalência de parasitos foi relativamente baixa na amostra quando comparada ao resultado de outro estudo realizado no estado de Santa Catarina (STALLIVIERE et al., 2009), o que permite inferir que a população de cães domiciliados em Chapecó está recebendo um cuidado adequado dos seus respectivos tutores. 0 conhecimento relatado pelos tutores pode explicar o cuidado verificado com os animais sob sua guarda. 
Os cães desempenham o papel de hospedeiro definitivo para algumas espécies de helmintos, que podem causar enfermidades importantes para o ser humano. Os resultados apontam para a necessidade de qualificar os canais e as informações disponibilizadas ao público sobre cuidados e o bem-estar animal, assim como o monitoramento das parasitoses que acometem animais domésticos.

\section{AGRADECIMENTOS}

O estudo foi apoiado pela Universidade Comunitária da Região de Chapecó (UNOCHAPECÓ), pelo Programa de Bolsas Universitárias de Santa Catarina (UNIEDU) e pelo município de Chapecó/SC setor de vigilância ambiental.

\section{REFERÊNCIAS}

ARAÚJO, J. V. Helmintoses intestinais em cães da microrregião de Viçosa, Minas Gerais. Rev Ceres, v. 53, n. 307, p. 363-365, 2015.

BARNABE, A. S. et al. Prevalência de parasitas intestinais em cães domiciliados na zona oeste da região metropolitana de São Paulo. Rev UNILUS, v. 12, n. 27, p. 28-31, 2015.

BARROS, B. A. F. et al. Ocorrência de parasitas gastrintestinais em fezes de cães coletadas em vias públicas do município de Valença - RJ. PUBVET, v. 12, n. 9, p. 1-9, 2018.

CHIEFFI, P. P. et al. Parasitoses intestinais - diagnóstico e tratamento. São Paulo: Lemos Editorial; 2002.

CHIEFFI, P. P.; NETO, V. A. Vermes, verminoses e a saúde pública. Ciên Cult, v. 55, n. 1, p. 41-43, 2003.

FEITOSA, F. L. F. Semiologia veterinária. São Paulo: Ed. Roca, 2016.

GENNARI, S. M. Principais helmintos intestinais em cães no Brasil. Bol Bayer Vet, v. 8, n. 2, p. 1-1, 2015.

FERRAZ, A. et al. Frequência de parasitos gastrintestinais, presentes em fezes de cães e gatos, analisadas no laboratório de doenças parasitárias da UFPEL, durante o ano de 2017. Sci An Health, v. 7, n. 1, p. 41-53, 2019.

GONZÁLEZ, A. C. et al. Prevalencia de parásitos intestinales zoonóticos en caninos (Canis lupus familiaris) del área urbana del municipio de Coyaima (Tolima). Rev Med, v. 23, n. 2, p. 24-34, 2015. 
KATAGIRI, S.; OLIVEIRA-SEQUEIRA, T. C. G. Zoonoses causadas por parasitas intestinais de cães e o problema do diagnóstico. Arq Inst Biol, v. 74, n. 2, p. 175-184, 2007.

LALLO, M. A. et al. Comportamento humano na criação de cães e a prevalência de parasitos intestinais com potencial zoonótico. Rev Acad Ciên An, v. 14, p. 119-128, 2016.

LIMA F. S. Contaminação ambiental por ovos de Ancylostoma spp. e Toxocara spp. em áreas de seis praças públicas do município de Valença, estado do Rio de Janeiro. Acta Biom Bras, v. 8, n. 1, p. 35-42, 2017.

MARMOL, A. B. Prevalência de ovos e larvas de Helmintos em amostras fecais de cães, coletadas em praças públicas do Município de Franca, SP, Brasil. Ev Cient Fund Ed Ituverava, v. 3, n. 3, p. 1-2, 2018.

MONTEIRO, S. G. Parasitologia na medicina veterinária. 2. ed. São Paulo: Editora Rocca, 2017. p. 15-29.

MOTTA, M. E. F. A.; SILVA, G. A. P. Diarreia por parasitas. Rev Bras Saúde Mat Inf, v. 2, n. 2, p. 117-127, 2002.

OLIVARI, M. B. D. et al. Avaliação do nível de conhecimento sobre zoonoses e guarda responsável e perfil dos proprietários de animais de estimação de um bairro no município de Jaboticabal/SP. ARS Vet, v. 31, n. 2, p. 21, 2015.

OLIVEIRA, A. C. et al. Ctenocephalides canis e Ctenocephalides felis: revisão de literatura. Rev Cient Eletr Med Vet, v. 11, p. 1-5, 2008.

RIBEIRO, C. M. et al. Infecções por parasitos gastrintestinais em cães domiciliados e suas implicações na transmissão zoonótica. Vet Zootec, v. 22, n. 2, p. 238-244, 2015;

RIBEIRO, V. M. Controle de helmintos de cães e gatos. XIII Congresso Brasileiro de Parasitologia Veterinária \& I Simpósio Latino-Americano de Ricketisioses, Ouro Preto, MG, 2004. Rev Bras Parasitol Vet, v. 13, suplemento 1, p. 88-95, 2004.

STALLIVIERE, F. M. et al. Ectoparasitos em Canis familiaris da cidade de Lages, SC, Brasil e aspectossócio-econômicos e culturais das famílias dos proprietários dos animais. Rev Ciên Agrovet, v. 8, n. 2, p. 179-183, 2009.

TRAVERSA, D. Are we paying too much attention to cardiopulmonary nematodes and neglecting oldfashioned worms like Trichuris vulpis? Paras Vectors, v. 4, n. 32, p. 1-11, 2011.

TROCCAP - Conselho Tropical para Parasitos de Animais de Companhia. Diretrizes para o diagnóstico, tratamento e controle de endoparasitos caninos nos trópicos. TroCCAP, 2017. 
URQUHART, G. M. et al. Parasitologia veterinária. 2. ed. Rio de Janeiro: Editora Guanabara Koogan, 1998.

VIEIRA, M. T. et al. Desenho da amostra e participação no Estudo Educatel. Cad Saúde Públ, v. 35, n. Suppl 1, e00167217, 2019.

YOSHINARI, N. H. et al. Doença de lyme-símile brasileira ou síndrome Baggio-Yoshinari: zoonose exótica e emergente transmitida por carrapatos. Rev Assoc Méd Bras, v. 56, n. 3, p. 363-369, 2010. 
1 Acadêmica do Curso de Medicina Veterinária, Universidade Comunitária da Região de Chapecó - Unochapeco, Chapecó/SC. E-mail: jessica.paula@unochapeco.edu.br

2 Acadêmica do Curso de Medicina Veterinária, Universidade Comunitária da Região de Chapecó - Unochapeco, Chapecó/SC. E-mail: veri.simon@hotmail.com

3 Acadêmica do Curso de Medicina Veterinária, Universidade Comunitária da Região de Chapecó - Unochapeco, Chapecó/SC. E-mail: darquila.piva@unochapeco.edu.br

4 Acadêmica do Curso de Medicina Veterinária, Universidade Comunitária da Região de Chapecó - Unochapeco, Chapecó/SC. E-mail: thaina.kemerich@unochapeco.edu.br

5 Acadêmica do Curso de Medicina Veterinária, Universidade Comunitária da Região de Chapecó - Unochapeco, Chapecó/SC. E-mail: damolinflavia1@gmail.com

6 Médico Veterinário; Coordenador do setor de Vigilância Ambiental em Saúde do município de Chapecó/SC.

E-mail: dmmfritzen@gmail.com

7 Doutor em Biodiversidade Animal pela Universidade Federal de Santa Maria - UFSM; Professor do Programa de Pós-Graduação em Ciências da Saúde da Universidade Comunitária da Região de Chapecó - Unochapecó, Chapecó/ SC.E-mail: junir@unochapeco.edu.br

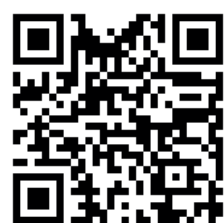

A autenticidade desse artigo pode ser conferida no site https://periodicos. set.edu.br

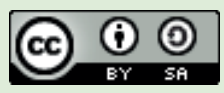

Este artigo é licenciado na modalidade acesso abertosob a Atribuição-Compartilhalgual CC BY-SA
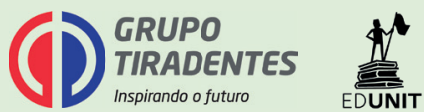